\title{
FACTORES Y AGENTES DINÁMICOS QUE GENERARON LA TEMPORADA NIVAL 2015 INTERRUMPIENDO LA SEQUÍA 2010-2014, EN LOS ANDES ÁRIDOS.
}

\author{
Dr. Poblete, Arnobio Germán \\ Profesor Titular exclusivo. \\ Inst. de Geografía Aplicada. Universidad Nacional San Juan. \\ Email: agpoblete@gmail.com \\ Prof. Valdez, Vanesa María \\ Profesor Ayudante de 1ra. \\ Inst. de Geografía Aplicada. Universidad Nacional San Juan. \\ Email: valdez.aldana75@gmail.com \\ Atencio, Maira Antonella. \\ Ayudante de 2da. \\ Email: atenciomaira@gmail.com
}

\section{RESUMEN}

La temporada nival de 2015 en los Andes Áridos, generó la interrupción de la última sequía que comenzó en el 2010, presentando una irregularidad que contradijo las condiciones de borde favorables desde abril, puesto que hasta el 4 de julio no se produjeron nevadas de importancia, revirtiéndose la situación significativamente, desde 12 de julio con un máximo entre 5 y 12 de agosto, debido a la presencia de: un fuerte evento "El Niño", un Anticiclón del Océano Pacífico Sur (ASPSO) debilitado y una Oscilación Decadal Pacífica (PDO) positiva, entre otros.

Este trabajo se propone describir estadísticamente esta temporada y explicitar los factores climatológicos dinámicos, oceánicos, circulación atmosférica regional y de la cupla océanoatmósfera que, concatenados, produjeron lo descripto.

Palabras Claves: temporada nival; sequía; condiciones de borde; factores climatológicos dinámicos.

\section{AGENTS AND DYNAMIC FACTORS THAT GENERATED THE SEASON 2015 NIVAL INTERRUPTING DROUGHT 2010-2014, IN ARID ANDES.}

\section{ABSTRACT}

The nival season in 2015 Arid Andes, generated the interruption of the last drought that began in 2010, presenting an irregularity that contradict the conditions favorable border starting on April, since July 4 until no significant snowfall occurred, reversing the situation significantly, from July 12 with a maximum between 5 and 12 August, due to the presence of: a strong "El Niño," a South Pacific anticyclone (ASPSO) weakened and Pacific Decadal Oscillation positive (PDO), among others.

This paper intends describe statistically this season and explain the dynamic climatological factors, oceanic, regional atmospheric circulation and ocean-atmosphere cupla, concatenated, produced as described

Key words: nival season; drought; edge conditions; dynamic climatological factors.

\section{INTRODUCCION}

La temporada nival de 2015 mostró una gran singularidad debido a que por su magnitud generó la inflexión de la sequía que se venía produciendo desde el 2010, además presentó una secuencia temporal muy irregular y en muchos casos contradiciendo las condiciones de borde favorables dadas

Publicado en formato digital: Dr. Arnobio Germán Poblete, Prof. Vanesa María Valdez y Maira Antonella Atencio. FACTORES Y AGENTES DINÁMICOS QUE GENERARON LA TEMPORADA NIVAL 2015 INTERRUMPIENDO LA SEQUÍA 2010-2014, EN LOS ANDES ÁRIDOS. Revista Geográfica Digital. IGUNNE. Facultad de Humanidades. UNNE. Año 13. № 25. Enero - Junio 2016. ISSN 1668-5180 Resistencia, Chaco. 
por un escenario hemisférico dominado por la presencia de un evento "Niño" muy significativo y pronunciado en el área 3+4 (SSTN3+4), (http://www.cpc.ncep.noaa.gov/products/international/, 2015) sumado a un anticiclón del Océano Pacífico Sur (ASPSO) debilitado y una Oscilación Decadal Pacífica (PDO) positiva (http://jisao.washington.edu/pdo/PDO.latest, 2015) condiciones que auguraban una precipitación nívea muy importante desde abril. A pesar de ello hasta el 4 de julio prácticamente no se produjo precipitación nival, situación que se revirtió el 12 de julio con una significativa nevada seguida por otra de mayor magnitud ocurrida entre el 5 y 12 de agosto, siendo esta la segunda más importante de la última década. Ramlot, M. J. P., (1970), Rutlant, W., (1989), Vorosmarty, C.J. et al. (2000)

Complementando lo descripto, se presenta un mes de octubre con caída de nieve inusual e inhibición de la ablación debida a una primavera anómala, con temperaturas inferiores a lo normal (Poblete y Valdez, 2015).

Este trabajo tiene como propósito describir estadísticamente esta temporada y explicitar los factores climatológicos dinámicos, oceánicos, circulación regional y de la cupla océano-atmósfera que, concatenados, produjeron lo descripto, y además explicar la irregularidad de los episodios níveos.

\section{DATOS Y MÉTODOS}

A causa de la insuficiencia de mediciones nivales que abarquen un período mayor que 100 años en los Andes Áridos (ver figuras 1 y 2), se emplean como índice para valuarlas los registros de los derrames anuales del río San Juan (DJUA) en el período 1909-2014, puesto que representa lo sucedido en la temporada nival correspondiente (Poblete, 2014). Los datos del DJUA fueron aportados por el Departamento de Hidráulica de la Provincia de San Juan y la Secretaría de Recursos Hídricos de la Nación (2014). Los registros de nieve de abril-septiembre fueron descargados de la estación Tascadero localizada en Chile a pocos km al oeste de la cuenca del río San Juan-Figura 2-.

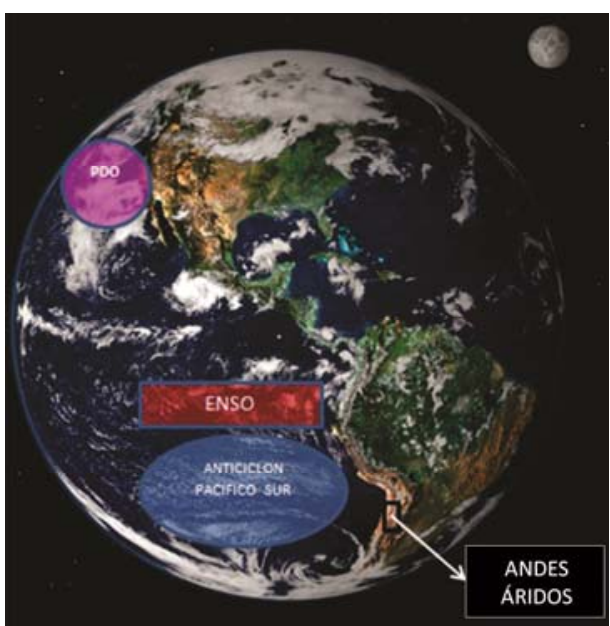

Figura 1: Ubicación del área de estudio y de los factores analizados en este trabajo. Elaboración propia.

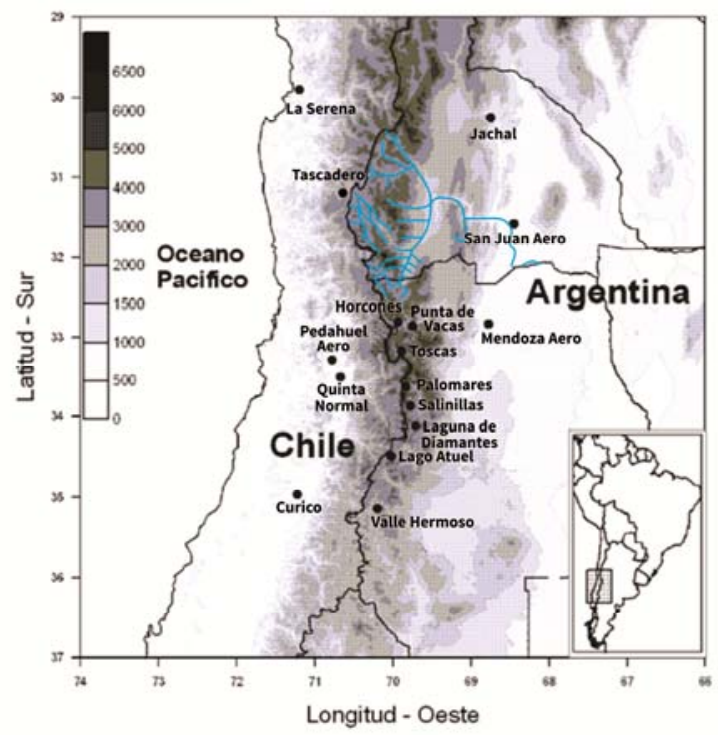

Figura 2: Ubicación del área de estudio y del índice que la representa en rojo (Rio San Juan-DJUA-). Adaptado de Viale, (2010).

Las SSTN3+4 fueron obtenidas del sitio http://www.cpc.ncep.noaa.gov/products/, los del ASPSO recopilados de diversas fuentes tales como La Dirección Meteorológica de Chile, Laboratorio

Publicado en formato digital: Dr. Arnobio Germán Poblete, Prof. Vanesa María Valdez y Maira Antonella Atencio. FACTORES Y AGENTES DINÁMICOS QUE GENERARON LA TEMPORADA NIVAL 2015 INTERRUMPIENDO LA SEQUÍA 2010-2014, EN LOS ANDES ÁRIDOS. Revista Geográfica Digital. IGUNNE. Facultad de Humanidades. UNNE. Año 13. № 25. Enero - Junio 2016. ISSN 1668-5180 Resistencia, Chaco. 
Climatológico Sudamericano sede NOAA y otras vía internet. Los de la PDO descargados del sitio http://jisao.washington.edu/pdo/.La ubicación aproximada de los mismos se muestra en la figura 1.a.

Se utilizaron las herramientas estadísticas usuales entre las que se destaca la variable estandarizada, que mide la desviación de cada dato respecto de la media en unidades de desviación típica.

La nueva variable: $z_{i}=\frac{x_{i}-\bar{X}}{S}$

Con $z_{i}$ la variable estandarizada obtenida de los $\mathrm{N}$ valores de $\mathrm{x}$ cuya media $(\bar{X})$ y desviación típica $(S)$, tienen una vez tipificadas, una media $=0 \quad(\bar{Z}=0)$ y desviación típica $=1 \quad\left(S_{z}=1\right)$. (Draper, et. al. 1976: 10)

Y además el Reanalysis del NCEP/NCAR para la detección de Isocorrelaciones espaciales.

\section{RESULTADOS Y DISCUSIÓN}

Debido al incipiente "Niño" se esperaba una temporada nival abundante desde abril. Sin embargo hasta el 4 de julio prácticamente no se produjeron nevadas, resultando muy irregular como se analizará a continuación (Poblete, 2015).

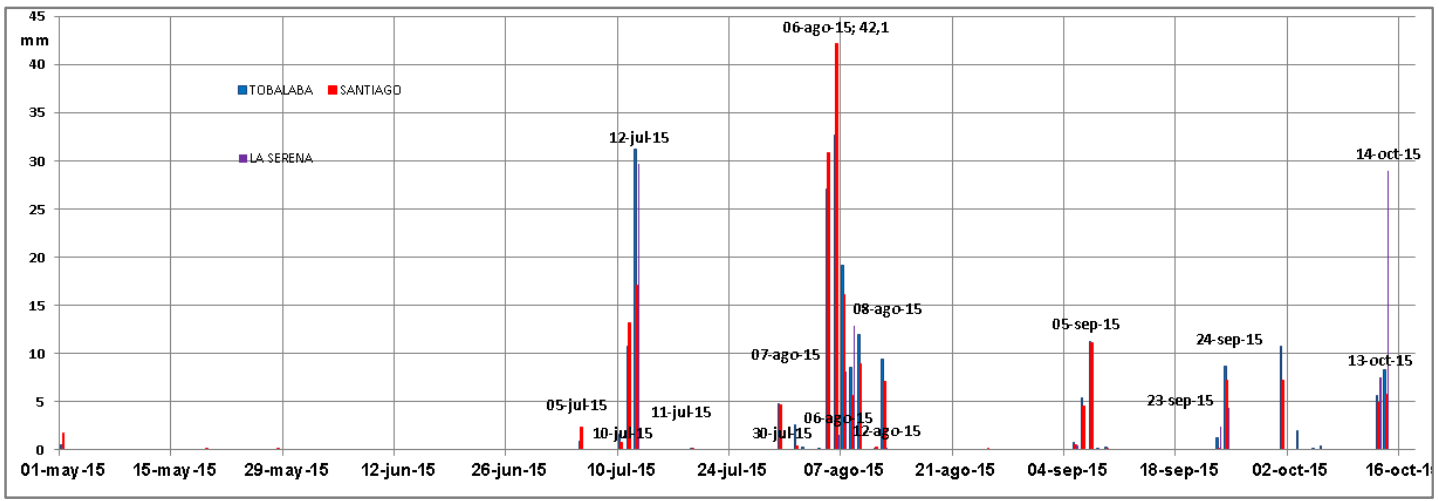

Figura 3: Eventos de precipitación nival ocurridos entre el 1 de mayo y el 16 de octubre 2015. (Datos de: DMC-

Dirección Meteorológica de Chile-, 2015)

La figura 3 muestra los eventos de precipitación nival ocurridos en la temporada analizada. Obsérvese que hasta el 4 de julio no se registraron nevadas (ver figuras 4 y 7 ), hecho que fue interrumpido por la primera de importancia acaecida el 12 de julio (ver figura 5 y 7 ), a la que le siguió la secuencia registrada entre el 5 y 8 de agosto que resultaron ser las más importantes del año (ver figura 6 y 7 ).

Publicado en formato digital: Dr. Arnobio Germán Poblete, Prof. Vanesa María Valdez y Maira Antonella Atencio. FACTORES Y AGENTES DINÁMICOS QUE GENERARON LA TEMPORADA NIVAL 2015 INTERRUMPIENDO LA SEQUÍA 2010-2014, EN LOS ANDES ÁRIDOS. Revista Geográfica Digital. IGUNNE. Facultad de Humanidades. UNNE. Año 13. № 25. Enero - Junio 2016. ISSN 1668-5180 Resistencia, Chaco.

En: http://hum.unne.edu.ar/revistas/geoweb/default.htm 


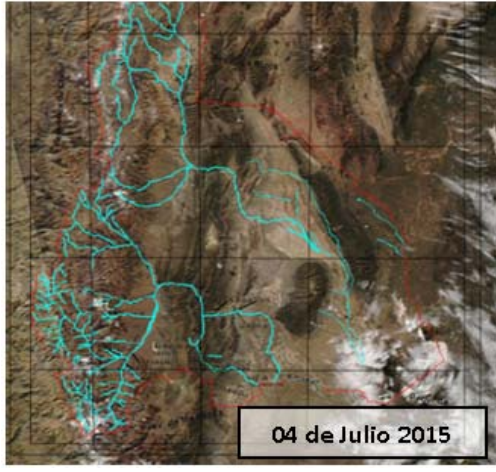

Figura 4: Estado de la cordillera hasta el 4 de julio de 2015.

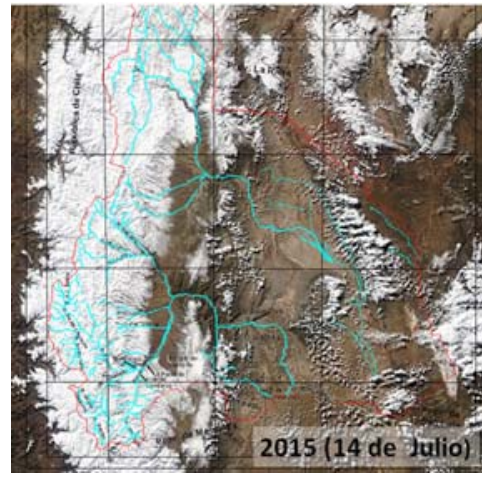

Figura 5: Estado de la cordillera después de la importante nevada del 12 de julio.

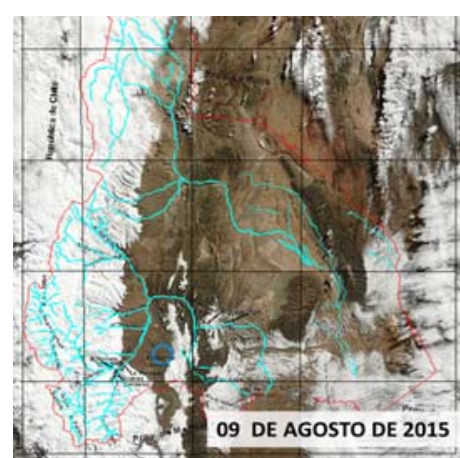

Figura 6: Estado de la cordillera después de la importante nevada del 5-8 de agosto.

Fuente: Elaboración propia sobre imágenes de NOAA-MODIS.-

La figura 7 muestra las nevadas registradas teniendo en cuenta su equivalente de agua de nieve (EAN) medida en la estación Horcones -ver figura 2- y sus similares de los últimos 10 años comparadas con la secuencia media (negro), y como complemento en la figura 8 se grafican los derrames anuales del río San Juan correspondientes. Obsérvese el contraste entre la temporada del $2005\left(2853 \mathrm{Hm}^{3}\right.$ ), la mayor de la década, y la del 2010 la menor $\left(890 \mathrm{Hm}^{3}\right)$. Gran parte de ellas tienen una cierta regularidad comenzando a principios de mayo, con un máximo entre junio, agosto para luego decrecer a fines de setiembre. Sin embargo la temporada 2015 se muestra totalmente disímil por su asimetría provocada por la ausencia de nevadas hasta el 4 de julio cuando realiza su primer escalón que luego es maximizado en los primeros días de agosto. Por otra parte, en contraste con el resto, se extiende con precipitaciones importantes hasta el 16 de octubre debido a una primavera anormalmente fría provocada por la activación de la corriente de Las Malvinas. También se observa en la figura 8 el pronóstico para la temporada 2015-16 que cuando fue calculado, solo teniendo en cuenta el lapso abril- septiembre, arrojó un derrame de $1660 \mathrm{Hm}^{3}$ pero por la relevancia de las nevadas anómalas del mes de octubre se estimó nuevamente dando por resultado $1780 \mathrm{Hm}^{3}$ estando en condiciones de alcanzar su media de $2051 \mathrm{Hm}^{3}$.

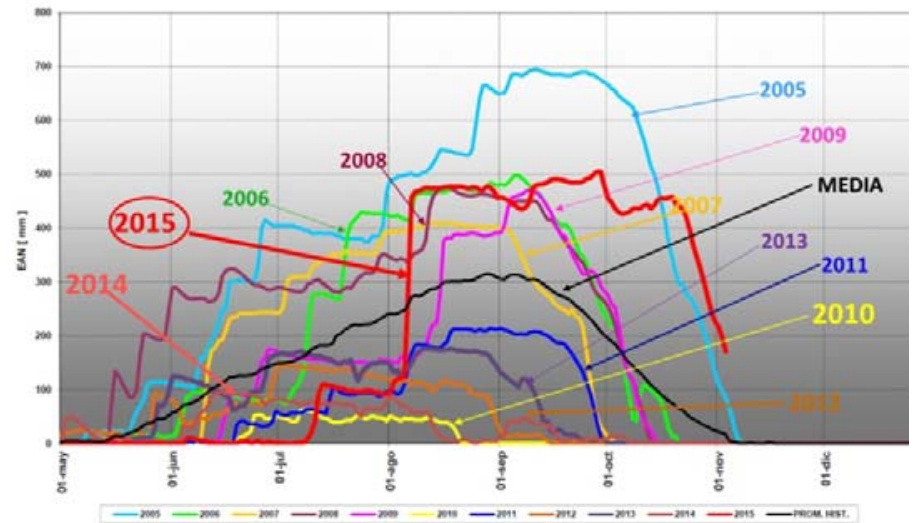

Figura 7: Equivalente de agua de nieve (EAN) de la temporada 2015 en la estación Horcones -ver figura 2- comparada con las registradas en la última década. Fuente DGI de Mendoza.

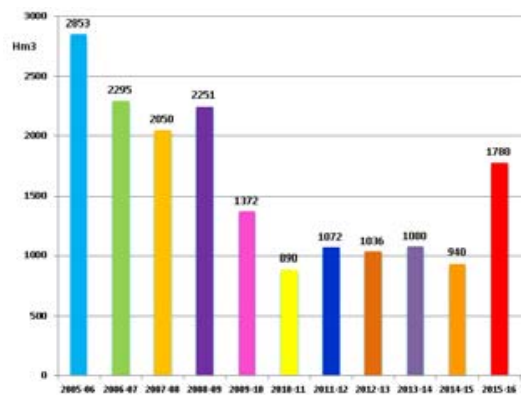

Figura 8: Derrames anuales del río San Juan correspondientes a las temporadas nivales mostradas en la figura 9.

Otro elemento relevante para ser tenido en cuenta en el pronóstico de los caudales estivales, es la primavera con una anomalía térmica que coadyuvó a que la ablación se retarde respecto de otros

Publicado en formato digital: Dr. Arnobio Germán Poblete, Prof. Vanesa María Valdez y Maira Antonella Atencio. FACTORES Y AGENTES DINÁMICOS QUE GENERARON LA TEMPORADA NIVAL 2015 INTERRUMPIENDO LA SEQUÍA 2010-2014, EN LOS ANDES ÁRIDOS. Revista Geográfica Digital. IGUNNE. Facultad de Humanidades. UNNE. Año 13. № 25. Enero - Junio 2016. ISSN 1668-5180 Resistencia, Chaco.

En: http://hum.unne.edu.ar/revistas/geoweb/default.htm 
años. También influyó en el retraso de las veranadas típicas de los departamentos de Iglesia y Calingasta y sus colaterales chilenos (Diario de Cuyo, 27 de nov. 2015).

\section{Factores que incidieron en la irregularidad de la temporada nival descripta}

Se decide explicar dinámicamente la temporada nival recién descripta, poniendo énfasis en su irregularidad, de ahí que se analicen las condiciones de borde imperantes antes y después del 4 de julio, dado que recién en esta fecha comenzaron a producirse las nevadas que por su magnitud aportaron a que la temporada de 2015 sea un punto de inflexión en la sequía que se producía desde el 2010 (Poblete, Valdez, 2015).

\section{Condiciones atmosférica antes del 4 de julio.-}

Debido a la importancia sobre la precipitación nival en los Andes Áridos de las SST en el área Niño $3+4$, se analiza su situación en el inicio de la temporada y a principios de junio.

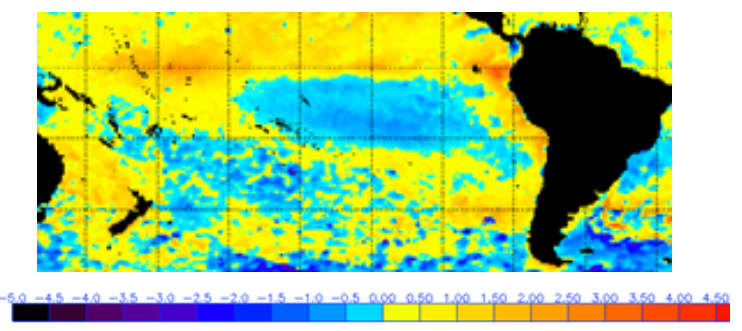

Figura 9: Anomalía de la temperatura superficial del mar en el día 9 de abril de 2015 .

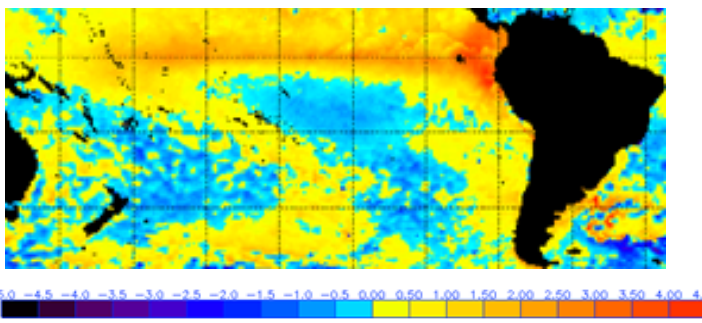

Figura 10: Anomalía de la temperatura superficial del mar en el día 01 de junio de 2015

Fuente: http://www.cpc.ncep.noaa.gov/products/international/, 2015.

En la figura 9 se observa que a principios de abril solamente en las áreas "Niño" $1+2$ y 4 se insinuaba un calentamiento que contribuyó a que el promedio del área citada sea de $0.96{ }^{\circ} \mathrm{C}$ (ver figura 11), en tanto que el primero de junio (figura 10 ) ya muestra un desarrollo mucho más considerable, incluyendo el área $3+4$, de esta manera el promedio se incrementa a $1,39^{\circ} \mathrm{C}$ por encima de lo normal (ver figura 11) Lo descripto insinúa que el Niño todavía entre esas fechas estaba en desarrollo, esto explicaría en parte que no haya habido nevadas de importancia hasta la mencionada fecha límite.

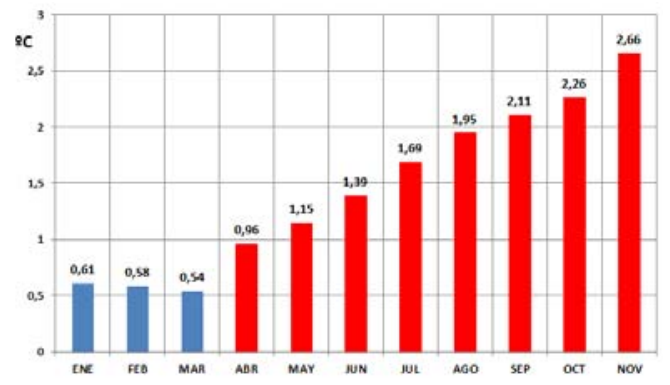

Figura 11: Evolución de las anomalías de las SST en el área Niño $3+4$. Calculadas como promedio en el área: $5 \mathrm{~S}-5 \mathrm{~N}$ y $170-120 \mathrm{~W}$. Desde enero a noviembre de 2015.

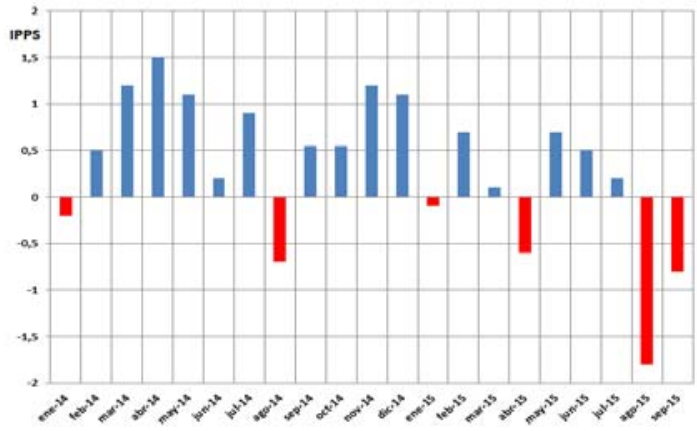

Figura 12: Evolución mensual del IPPS índice que mide la actividad del ASPSO. Enero del 2014 a setiembre del 2015. (Datos de: DMC- Dirección Meteorológica de Chile-, 2015)

Según el IPPS (ver figura 12) el ASPSO estaba potenciado en los meses de mayo, junio y julio hecho que contribuyó a la ausencia de precipitaciones de importancia, para confirmarlo se analizan los campos de presión en superficie, $500 \mathrm{mb}$ y $200 \mathrm{mb}$, en el mes de junio como representante de este lapso.

Publicado en formato digital: Dr. Arnobio Germán Poblete, Prof. Vanesa María Valdez y Maira Antonella Atencio. FACTORES Y AGENTES DINÁMICOS QUE GENERARON LA TEMPORADA NIVAL 2015 INTERRUMPIENDO LA SEQUÍA 2010-2014, EN LOS ANDES ÁRIDOS. Revista Geográfica Digital. IGUNNE. Facultad de Humanidades. UNNE. Año 13. № 25. Enero - Junio 2016. ISSN 1668-5180 Resistencia, Chaco. 


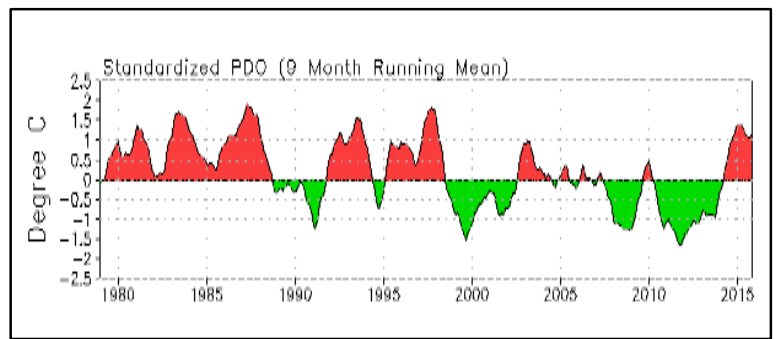

Figura 13: Evolución mensual del índice que mide la variabilidad PDO. 1980 - 2015, http://jisao.washington.edu/pdo/.

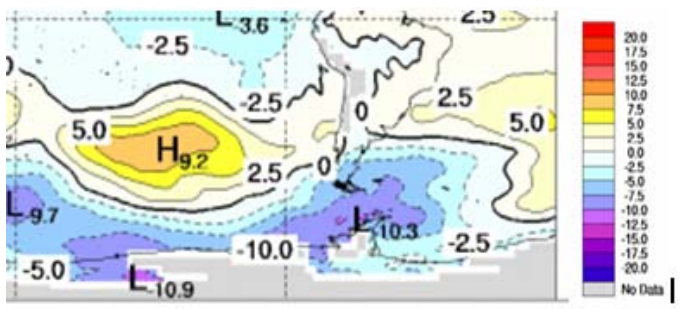

Figura 14: Presión atmosférica a nivel del mar en junio. Boureau of Climatology the Australia

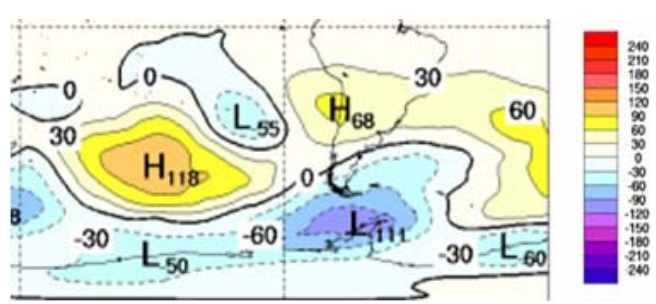

Figura 15: Geopotencial de los $500 \mathrm{mb}$ en junio.

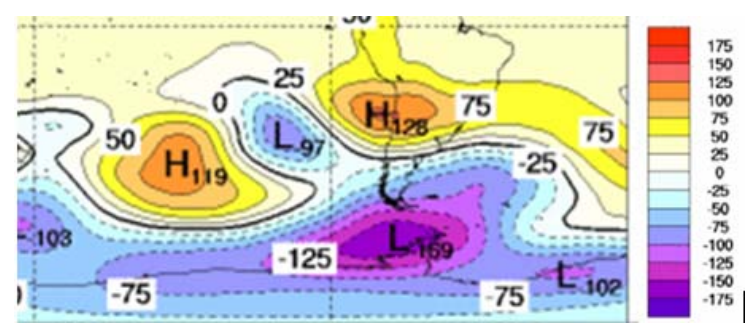

Figura 16: Geopotencial de los $200 \mathrm{mb}$ en junio.

Fuente: http://www.bom.gov.au/cgi, 2015.

La figura 14 muestra el campo de presión media en superficie del mes de junio obsérvese una condición sin anomalías significativa del ASPSO cercanas a la costa salvo un ápice próximo a Chile central y un núcleo potenciado mar adentro, condiciones que no contribuyen a que el paso de los agentes precipitantes sea fluido. Esta condición de bloqueo se maximiza en los geopotenciales de los $500 \mathrm{mb}$ (ver figura 15) y $200 \mathrm{mb}$ (ver figura 16), con anomalías positivas muy significativas teniendo en cuenta que en esas alturas es donde se produce la precipitación nival.

Sin embargo la PDO está en la fase positiva que se inicia en 2014 (ver figura 13).

Publicado en formato digital: Dr. Arnobio Germán Poblete, Prof. Vanesa María Valdez y Maira Antonella Atencio. FACTORES Y AGENTES DINÁMICOS QUE GENERARON LA TEMPORADA NIVAL 2015 INTERRUMPIENDO LA SEQUÍA 2010-2014, EN LOS ANDES ÁRIDOS. Revista Geográfica Digital. IGUNNE. Facultad de Humanidades. UNNE. Año 13. № 25. Enero - Junio 2016. ISSN 1668-5180 Resistencia, Chaco.

En: http://hum.unne.edu.ar/revistas/geoweb/default.htm 


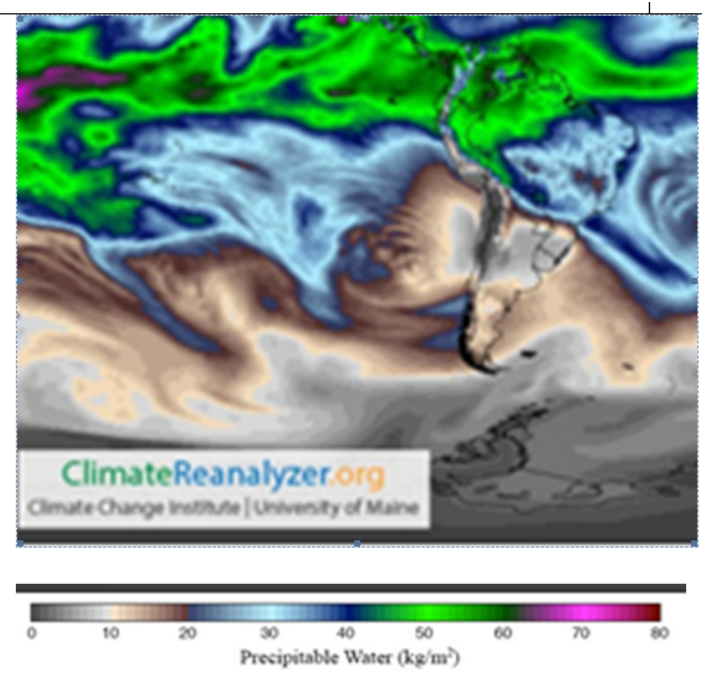

Figura 17: Agua precipitable en superficie medida en $\mathrm{kg} / \mathrm{m}^{2}$ en junio de 2015 .

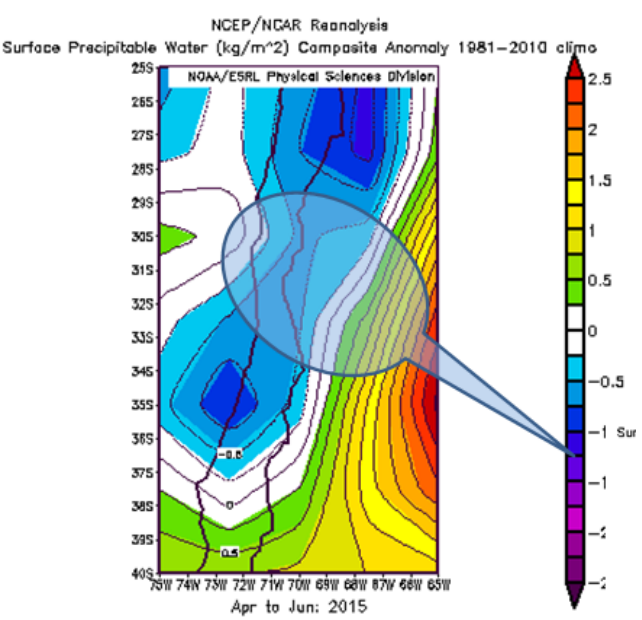

Figura 18: Agua precipitable en superficie medida en $\mathrm{kg} / \mathrm{m}^{2}$ en el periodo abril-junio de 2015. Fuente: Reanalysis NCEP/NCAR

En la figura 17 se observa la disponibilidad hemisférica del agua precipitable correspondiente al mes de junio de 2015, la mayor concentración se encuentra en las proximidades de la línea de convergencia intertropical ITCZ y una moderada cantidad en el oeste del océano Pacífico haciéndose nula en el entorno de los Andes Áridos, Chile Central y mar adyacente. Hecho que se extiende a todo el periodo abril junio como se muestra en la figura 18.

Lo descripto respecto del comportamiento de las SST en el área 3+4, de los centros de presión en los distintos niveles y de la disponibilidad del agua precipitable explica la ausencia de precipitaciones durante el periodo abril- junio. Puesto que no estuvieron presentes la condición necesaria (humedad en la atmósfera) y suficiente (agentes precipitantes) para que haya precipitación.

\section{Condiciones atmosféricas después del 4 de julio.}

Dado que, como se dijo, el 4 de julio marco un punto de ruptura entre la ausencia de nevadas y el inicio de las precipitaciones significativas, se analizan las condiciones oceánicas y atmosféricas imperantes desde esa fecha hasta el final de la temporada.

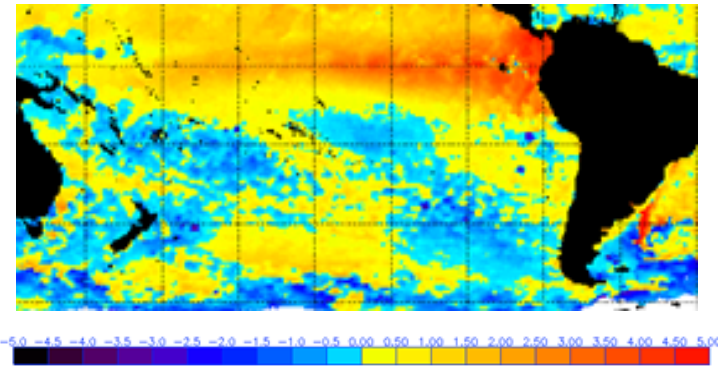

Figura 19: Anomalía de la temperatura superficial del mar en el día 6 de agosto de 2015

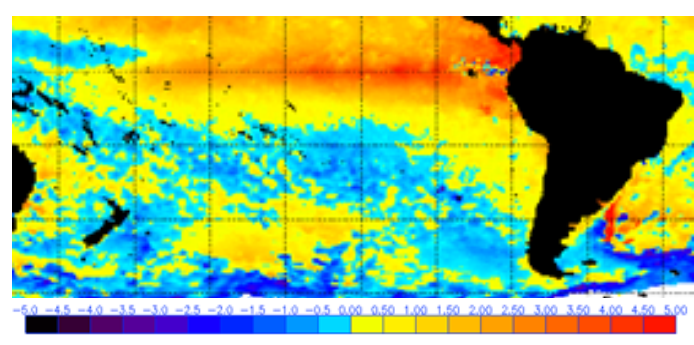

Figura 20: Anomalía de la temperatura superficial del mar en el día 10 de setiembre de 2015

La anomalía de las SST en el día 6 de agosto muestran ya un Niño muy desarrollado con casi $2{ }^{\circ} \mathrm{C}$ por encima de lo normal (ver figura 19) consolidándose en el mes de setiembre con un área de

Publicado en formato digital: Dr. Arnobio Germán Poblete, Prof. Vanesa María Valdez y Maira Antonella Atencio. FACTORES Y AGENTES DINÁMICOS QUE GENERARON LA TEMPORADA NIVAL 2015 INTERRUMPIENDO LA SEQUÍA 2010-2014, EN LOS ANDES ÁRIDOS. Revista Geográfica Digital. IGUNNE. Facultad de Humanidades. UNNE. Año 13. № 25. Enero - Junio 2016. ISSN 1668-5180 Resistencia, Chaco.

En: http://hum.unne.edu.ar/revistas/geoweb/default.htm 
calentamiento más extendida (ver figura 20) y una anomalía positiva de $2,11^{\circ} \mathrm{C}$ por encima de lo normal.

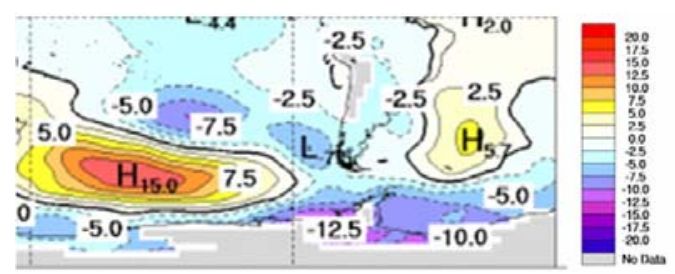

Figura 21: Presión atmosférica media mensual a nivel del mar en agosto de 2015.

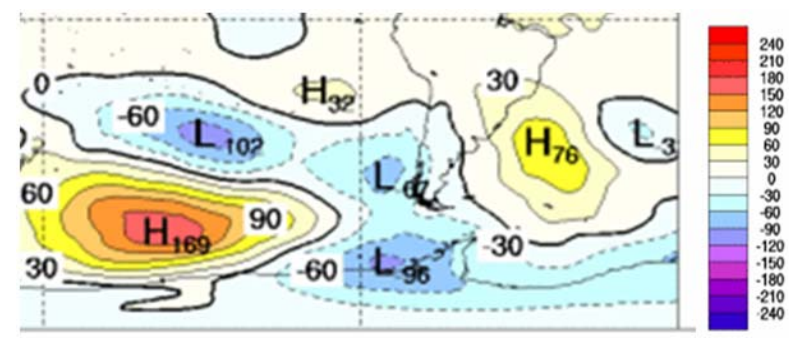

Figura 22: Altura media mensual del geopotencial de los $500 \mathrm{mb}$ en agosto de 2015.

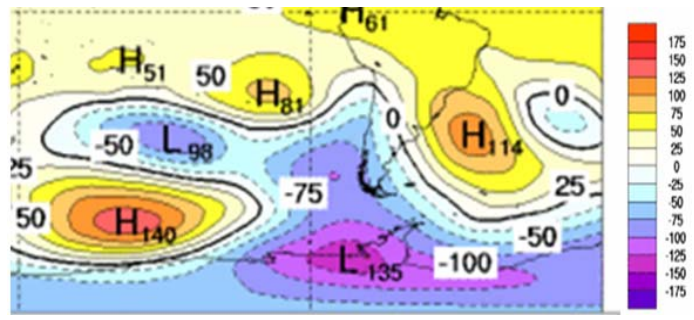

Figura 23: Altura media mensual del geopotencial de los $200 \mathrm{mb}$ en agosto de 2015. Fuente: http://www.bom.gov.au/cgi, 2015.

La figura 12 muestra que el IPPS es negativo en agosto y setiembre, indicando un anticiclón debilitado. La distribución areal de ese debilitamiento se puede observar en las figuras: 21 (anticiclón en superficie despresurizado), 22 (geopotencial de los $500 \mathrm{mb}$ con una baja presión dominante) y 23 (geopotencial de los $200 \mathrm{mb}$ con una significativa vaguada). Factores que facilitan al advenimiento de agentes precipitantes tales como vaguadas, bajas segregadas y frentes en superficie entre otros. En todos los casos se toma como muestra lo ocurrido en el mes de agosto puesto que fue el que mayor precipitación nívea recibió (ver figura 3). También la fase caliente de la PDO, en este caso, (ver figura13) influyó positivamente en el advenimiento de nevadas.

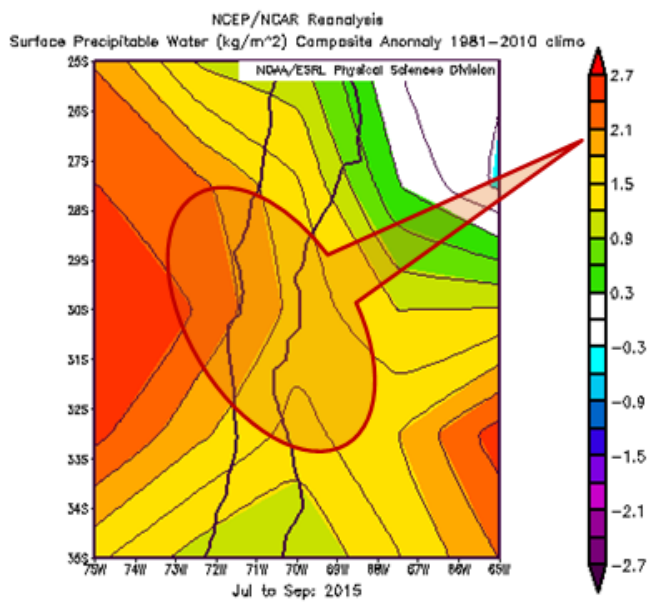

Figura 24: Agua precipitable en superficie medida en $\mathrm{kg} / \mathrm{m}^{2}$ en el periodo julio-septiembre de 2015. Reanalysis NCEP/NCAR

Publicado en formato digital: Dr. Arnobio Germán Poblete, Prof. Vanesa María Valdez y Maira Antonella Atencio. FACTORES Y AGENTES DINÁMICOS QUE GENERARON LA TEMPORADA NIVAL 2015 INTERRUMPIENDO LA SEQUÍA 2010-2014, EN LOS ANDES ÁRIDOS. Revista Geográfica Digital. IGUNNE. Facultad de Humanidades. UNNE. Año 13. № 25. Enero - Junio 2016. ISSN 1668-5180 Resistencia, Chaco.

En: http://hum.unne.edu.ar/revistas/geoweb/default.htm 
Como la condición necesaria para que haya precipitación es la existencia de humedad en la atmósfera, se analiza la disponibilidad de agua precipitable observándose en la figura 24 que hubo un superávit en el periodo julio-setiembre promoviendo la ocurrencia de eventos níveos más frecuentes.

Teniendo en cuenta lo anterior, se analiza de manera particular el origen del agua precipitable que hizo posible las nevadas del 12 de julio y 5 al 8 de agosto, las más significativas de toda la temporada (ver figura 3,5 y 6). Para lo cual se introduce el concepto "río atmosférico" (Ralph et al., 2004) y su asociación con la Corriente en Chorro (Jet Stream) estimada en este caso con el viento zonal en $250 \mathrm{mb}$.

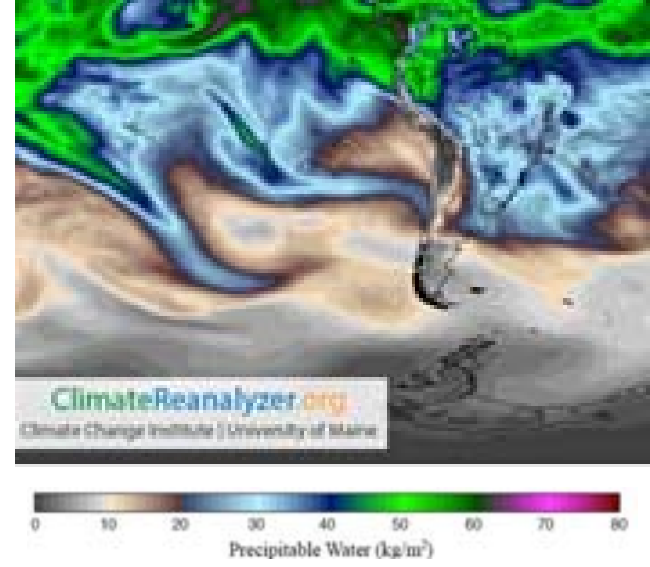

Figura 25: "Rio Atmosférico" que transporta el agua precipitable desde las regiones ecuatoriales hasta los Andes Aridos en el día 12 de julio. Fuente: ClimateReanalyzer.org

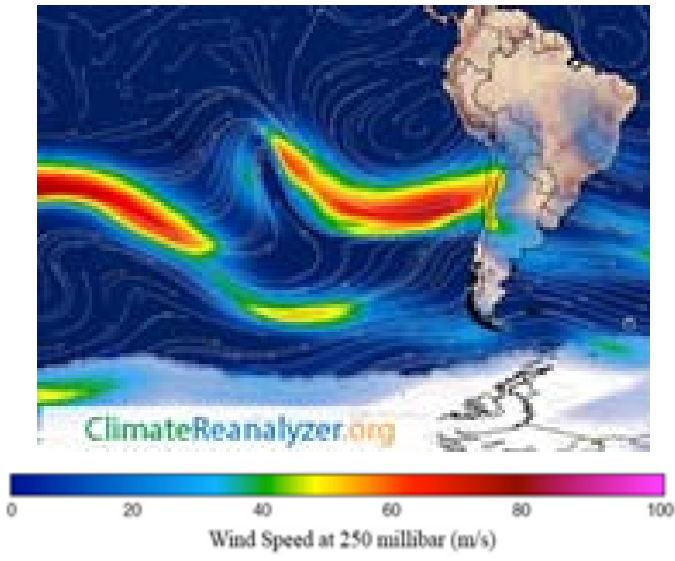

Figura 26: Comportamiento de la Corriente en Chorro estimada por la velocidad del viento zonal en los 250 $\mathrm{mb}$, en el día 12 de julio. Fuente: ClimateReanalyzer.org

En la figura 25 se observa el flujo de agua precipitable desde las regiones ecuatoriales hasta los Andes Áridos transportada por el citado "rio atmosférico", que resulta de estructuras contenidas en campos de viento intenso $\left(>12.5 \mathrm{~ms}^{-1}\right)$ con una cantidad de vapor de agua en columna vertical integrada superior a los $2 \mathrm{~cm}$. (Ralph and Dettinger, 2011) que sería producido por la inflexión de la Corriente en Chorro en esa latitud (figura 26).

Una situación semejante a la anterior se produjo el día 8 de agosto con un flujo de agua precipitable desde el Ecuador hasta los Andes Áridos (figura 27) posiblemente inducido, como se dijo, por la inflexión de la Corriente en Chorro (figura 28).

Publicado en formato digital: Dr. Arnobio Germán Poblete, Prof. Vanesa María Valdez y Maira Antonella Atencio. FACTORES Y AGENTES DINÁMICOS QUE GENERARON LA TEMPORADA NIVAL 2015 INTERRUMPIENDO LA SEQUÍA 2010-2014, EN LOS ANDES ÁRIDOS. Revista Geográfica Digital. IGUNNE. Facultad de Humanidades. UNNE. Año 13. № 25. Enero - Junio 2016. ISSN 1668-5180 Resistencia, Chaco.

En: http://hum.unne.edu.ar/revistas/geoweb/default.htm 


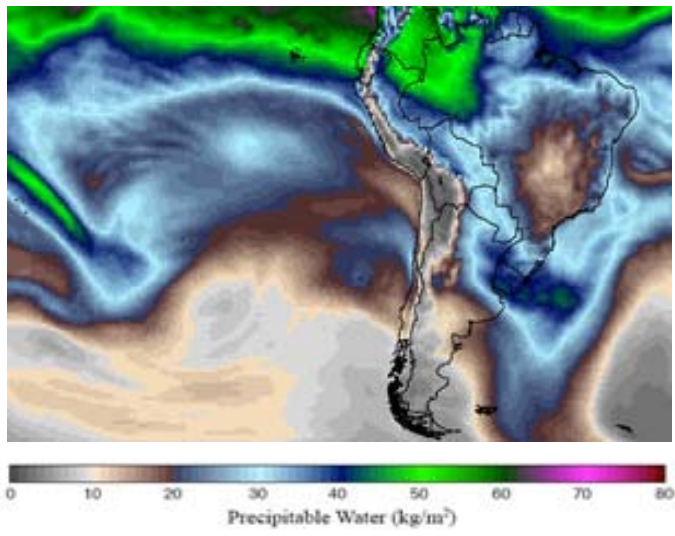

Figura 27: "Rio atmosférico" que transporta el agua precipitable desde las regiones ecuatoriales hasta los Andes Áridos en el día 8 de agosto. Fuente: ClimateReanalyzer.org

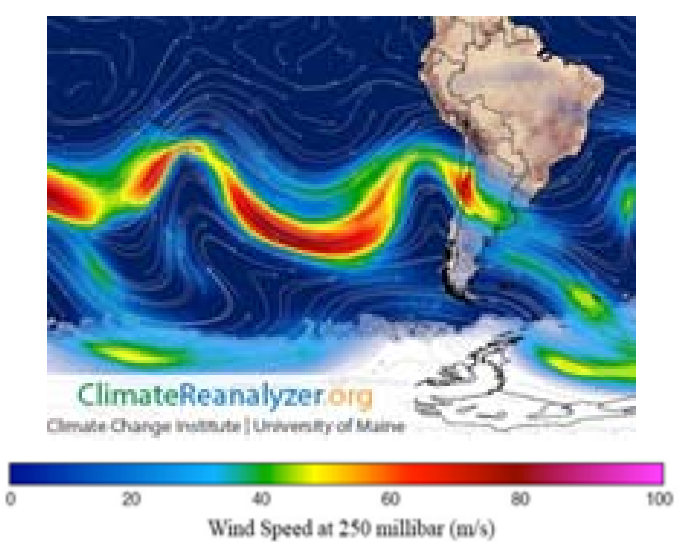

Figura 28: Comportamiento de la Corriente en Chorro estimada por el viento zonal a los $250 \mathrm{mb}$, en el día 8 de agosto. Fuente: ClimateReanalyzer.org

\section{CONCLUSIONES}

En la temporada nival de 2015, debido al incipiente "Niño" que se manifestó desde marzo, se esperaba que fuera con abundante caída nieve desde abril, sin embargo fue muy irregular puesto que hasta el 4 de julio no se produjeron nevadas significativas, ocurriendo la primera de importancia el 12 de julio, a la que le siguió una secuencia entre el 5 y 8 de agosto que resultaron ser las más importantes del año.

Del análisis dinámico se determinó que antes del 12 de julio se verificó lo siguiente: 1) a principios de abril solamente en las áreas "Niño" 1+2 y 4 se insinuaba un incipiente calentamiento que a principios de junio mostró un desarrollo más considerable, incluyendo el área $3+4$, esto explicaría en parte que no haya habido nevadas de importancia hasta la mencionada fecha límite.

El ASPSO estaba potenciado en los meses de mayo, junio y julio hecho que contribuyó a la ausencia de precipitaciones de importancia, siendo su distribución espacial sin anomalías significativas. Esta condición de bloqueo se maximiza en los geopotenciales de los 500 y $200 \mathrm{mb}$ con anomalías positivas muy significativas teniendo en cuenta que en esas alturas es donde se produce la precipitación nival. Sin embargo la PDO está en la fase positiva que se inicia en 2014

La disponibilidad hemisférica del agua precipitable correspondiente al mes de junio de 2015, tiene una mayor concentración en las proximidades de la línea de convergencia intertropical ITCZ haciéndose nula en el entorno de los Andes Áridos, Chile Central y mar adyacente. Hecho que se extiende a todo el periodo abril junio. Como se aprecia estuvieron ausentes la condición necesaria (humedad en la atmósfera) y suficiente (agentes precipitantes) para que haya precipitación.

Del análisis de las condiciones atmosféricas y oceánicas después del 4 de julio, se comprueba que: La anomalía de las SST a principios de agosto muestran ya un Niño muy desarrollado con casi $2{ }^{\circ} \mathrm{C}$ por encima de lo normal consolidándose en el mes de setiembre con un área de calentamiento más extendida y una anomalía positiva de $2,11^{\circ} \mathrm{C}$ por encima de lo normal.

EI IPPS es negativo especialmente en agosto y setiembre, indicando un anticiclón debilitado. Su distribución areal muestra un: anticiclón en superficie despresurizado, el geopotencial de los $500 \mathrm{mb}$ con una baja presión dominante y el de los $200 \mathrm{mb}$ con una significativa vaguada. Factores que facilitan al advenimiento de agentes precipitantes tales como vaguadas, bajas segregadas y frentes

Publicado en formato digital: Dr. Arnobio Germán Poblete, Prof. Vanesa María Valdez y Maira Antonella Atencio. FACTORES Y AGENTES DINÁMICOS QUE GENERARON LA TEMPORADA NIVAL 2015 INTERRUMPIENDO LA SEQUÍA 2010-2014, EN LOS ANDES ÁRIDOS. Revista Geográfica Digital. IGUNNE. Facultad de Humanidades. UNNE. Año 13. № 25. Enero - Junio 2016. ISSN 1668-5180 Resistencia, Chaco. 
en superficie entre otros. También la fase caliente de la PDO influyó positivamente en el advenimiento de nevadas.

La disponibilidad de agua precipitable tuvo un superávit en el periodo julio- setiembre, analizando el origen de la misma en las nevadas del 12 de julio y 5 al 8 de agosto, se introduce el concepto "río atmosférico" y su asociación con la Corriente en Chorro. El flujo de aquella desde las regiones ecuatoriales hasta los Andes Áridos se canalizó por un "rio atmosférico", que sería producido por la inflexión de la Corriente en Chorro en esa latitud. Una situación semejante a la anterior se produjo el día 8 de agosto.

Todas estas condiciones concatenadas dieron lugar a las abundantes nevadas registradas a partir del 12 de julio.

\section{AGRADECIMIENTOS}

Se agradece a la Universidad Nacional de San Juan por sus aportes económicos en el desarrollo de este trabajo y "Análisis de las Condiciones de Borde, Factores y Agentes Oceánicas de Circulación Atmosférica Regional y de la Cupla-Océano Atmósfera que Inciden en las Nevadas de los Andes Áridos.". CICITCA-UNSJ.

Publicado en formato digital: Dr. Arnobio Germán Poblete, Prof. Vanesa María Valdez y Maira Antonella Atencio. FACTORES Y AGENTES DINÁMICOS QUE GENERARON LA TEMPORADA NIVAL 2015 INTERRUMPIENDO LA SEQUÍA 2010-2014, EN LOS ANDES ÁRIDOS. Revista Geográfica Digital. IGUNNE. Facultad de Humanidades. UNNE. Año 13. № 25. Enero - Junio 2016. ISSN 1668-5180 Resistencia, Chaco.

En: http://hum.unne.edu.ar/revistas/geoweb/default.htm 
Revista Geográfica Digital. IGUNNE. Facultad de Humanidades. UNNE. Año 13. Nº 25. Enero - Junio 2016. ISSN 1668-5180 Resistencia, Chaco

\section{BIBLIOGRAFÍA}

- Climate Prediction Center National Weather. http: //www.cpc.ncep.noaa.gov /products/ international/, 2015.

- Jisao, Joint Institute For The Study Of The Atmosphere And Ocean. http: //jisao. washington. edu/pdo/.

- Poblete, A, G., Valdez V. M. 2015. Causas de la primavera anómala en 2015. Revista de Geografía UNC. Boletín de Estudios Geográficos Instituto de Geografía Facultad de Filosofía y Letras Universidad Nacional de Cuyo. ISSN 0374-6186. ISSN-L 0374-6186.-

- Poblete, A, G., Minetti, J. L. 2015. Identificación de Ondas Coherentes entre las Nevadas en Los Andes Áridos de Argentina-Chile y los Principales Factores Climáticos que las Condicionan. - Revista de Geofísica Vol. I.P.G.H- OEA.- ISSN: 0252-9769. México.

- Poblete, A, G., Valdez V. M. 2015. Análisis de la Temporada Nival 2015 que marcó un punto de inflexión en la Sequía en los Andes Áridos iniciada en 2010. Rev. de Geografía UNSJISSN 1514-1942- Vol. 20- № 20. San Juan. Argentina.

- Poblete, A. G., Aguiar, L. A. 2015. Relación Entre los Factores de Circulación Atmosférica Regional y de la Cupla Océano-Atmósfera con los Derrames Extremos de los Ríos San Juan Y Mendoza. Revista de Geografía UNC. Boletín de Estudios Geográficos Instituto de Geografía Facultad de Filosofía y Letras Universidad Nacional de Cuyo. ISSN 0374-6186. ISSNL 0374-6186.-

- Poblete, A.G. Pérez M. del V. 2015. Disponibilidad Del Recurso Hídrico Como Base De Cualquier Ordenamiento Territorial En Los Oasis Cuyanos. Jornadas de Ordenamiento Territorial. PRODEA. San Juan. Argentina. El 26- 27 de noviembre de 2015.

- Ralph F. M. And Dettinger M. D. 2011. Historical and National Perspectives on Extreme West Coast Precipitation Associated with Atmospheric Rivers during December 2010. NOAA/ESRL, U.S. Geological Survey, Scripps Institution of Oceanography, Physical Sciences Division, Boulder, Colorado. La Jolla, California

- Ramlot, M. J. P., 1970. Hacia el porvenir de la región cuyana. Mendoza, Universidad Nacional de Cuyo. Pág. 261.

- Rutlant, W., 1989. Variabilidad intraestacional e interanual en regiones extratropicales. Curso de variabilidad climática y su impacto ambiental. Departamento de meteorología. UBA. Buenos Aires. Pág. 345.

- Subsecretaría de Recursos Hídricos (2010) http://www.hidricosargentina.gov.ar/

- Viale, M., 2010. Características de las Precipitaciones Orográficas de invierno sobre los Andes Subtropicales Centrales. Universidad de Buenos Aires. Facultad de Ciencias Exactas y Naturales. Departamento de Ciencias de la Atmósfera y los Océanos. Tesis Doctoral.

Publicado en formato digital: Dr. Arnobio Germán Poblete, Prof. Vanesa María Valdez y Maira Antonella Atencio. FACTORES Y AGENTES DINÁMICOS QUE GENERARON LA TEMPORADA NIVAL 2015 INTERRUMPIENDO LA SEQUÍA 2010-2014, EN LOS ANDES ÁRIDOS. Revista Geográfica Digital. IGUNNE. Facultad de Humanidades. UNNE. Año 13. № 25. Enero - Junio 2016. ISSN 1668-5180 Resistencia, Chaco.

En: http://hum.unne.edu.ar/revistas/geoweb/default.htm 